\section{ARQUEOLOGÍA DE LA INFANCIA: UNA PERSPECTIVA ETNOARQUEOLÓGICA}

\author{
ARCHAEOLOGY OF CHILDHOOD: \\ AN ETHNOARCHAEOLOGICAL \\ PERSPECTIVE
}

GUSTAVO G. POLITIS (*)

\section{RESUMEN}

La producción material de los niños no ha sido habitualmente considerada en el análisis del registro arqueológico de los cazadores-recolectores, a pesar de que los datos etnográficos muestran que los niños son generadores importantes de cultura material, especialmente en los campamentos residenciales. En este trabajo se resume la información obtenida entre el grupo indígena Nukak, de la Amazonía colombiana, respecto a la participación infantil en la producción de objetos. A partir de esto, y de información de otros grupos cazadores-recolectores, se derivan expectativas arqueológicas que se confrontan con los materiales de los sitios arqueológicos de la región Pampeana de Argentina. Se propone una metodología para la identificación de la actividad infantil en los contextos arqueológicos, como un primer paso para discutir la agencia de actores sociales diversos en las sociedades del pasado.

\begin{abstract}
The material production of children has not usually been considered in the analysis of the hunter-gatherer archaeological record. However, ethnographic data shows that children are significant producers of material culture, especially in residential camps. In this paper, information about the activity of children among the Nukak from the Colombian Amazon, is summarized. Based on this, and on
\end{abstract}

(*) CONICET-INCUAPA Universidad del Centro (UNC) y Universidad de La Plata (UNLP). Facultad de Ciencias Sociales, Del Valle 5737, (7400) Olavarría, Argentina.

El artículo fue remitido en su versión final el 7-VIII-98. data from other hunter-gatherers, archaeological expectations are generated and compared with the archaeological record of the Pampean Region of Argentina. A methodology for the identification of children's activity is proposed as a first step towards the discussion of diversity in the agency of social actors in past societies.

Palabras claves: Etnoarqueología. Arqueología de la infancia. Cazadores-recolectores. América del Sur.

Key words: Ethnoarchaeology. Archaeology of childhood. Hunter-gatherers. South America.

\section{INTRODUCCIÓN}

En términos generales, en la interpretación tanto de los sitios de cazadores-recolectores, como de otro tipo de sociedades más complejas, se asume que los restos materiales recuperados (fundamentalmente los elementos tecnológicos y los desechos derivados de su confección y uso) han sido producidos por adultos. Los agentes son adultos por ausencia (default) de otros actores sociales. A partir de esta asunción implícita se han realizado inferencias sobre las conductas del pasado y se han propuesto modelos de diverso orden referentes a organización tecnológica, secuencia de producción y uso, maximización de materia prima, área de descarte, etc. Dentro de este marco, la forma, tecnología de producción y el tamaño de algunos artefactos (por ej. puntas de proyectil) son considerados como una expresión idiosincrásica y utilizados para la reconstrucción histórico-cultural o como una medida de 
variabilidad y distancia cultural. En estas reconstrucciones, basadas en supuestos agentes adultos, los niños no han sido visualizados como actores sociales y su producción material no se ha tenido en cuenta en el estudio arqueológico de los grupos cazadores-recolectores. Hay algunas excepciones que han propuesto que determinados objetos, pinturas rupestres de manos, o conjuntos artefactuales, especialmente líticos, fueron confeccionados o usados por niños (por ejemplo Frison, 1970; Gradin, 1981-1982; Bodú et alii, 1990; Fisher, 1990; Dawe, 1997; Park, 1998). En términos generales, el origen infantil se distinguía por "...the game-like and nonutilitarian character of poorly knapped pieces, displaying less skill than novice" (Finlay, 1997: 207). A pesar de estos ejemplos aislados, casi no se han desarrollado programas de investigación dirigidos a identificar y evaluar sistemáticamente la producción infantil dentro del registro arqueológico (para excepciones ver Dawe, 1997; Park, 1998) y a examinar las connotaciones socioculturales de esto.

Esto ha llevado a que los niños hayan sido recientemente equiparados con las mujeres, ya que, como consecuencia del sesgo adulto masculino, ambos han sido "invisibles" en la reconstrucción de las sociedades del pasado (Baker, 1997:187). Los intentos actuales para identificar la conducta infantil dentro de la arqueología surgen como respuesta al reconocimiento de esta situación, y como una continuación natural de la cada vez más intensa investigación de la arqueología del género (i.e Conkey y Spector, 1984; Gero y Conkey, 1991; Claassen, 1992; Scott,, 1997). Las similitudes de la feminidad y la infancia han sido también señaladas porAmorós (1997: 279): “[ambas] se nos muestran de este modo como dos reductos estamentales de la modernidad, según los cuales se configuró el espacio privado de la familia. Con todo, la infancia aparece como una característica adscriptiva temporal; la feminidad lo es de por vida". Debe recordarse que el concepto de infancia de nuestra sociedad es un producto moderno ya que fue Rousseau quien desarrolló una ideología alrededor de la niñez y de la "pureza" e "inocencia" infantil (Amorós, 1997: 276-279).

El naciente interés en examinar la participación infantil en la cultura material es también una derivación de la consideración de los niños como actores sociales significativos (James y Prout, 1990). Dentro del campo de la antropología social se ha planteado también esta sub-representación infantil y el hecho de que casi siempre son incorporados al análisis como parte de la esfera doméstica de los adultos, pero no como casos de estudio en sí mismo (Aguilar, 1994: 29).

En un trabajo anterior comencé a plantear que los niños (de aquí en adelante, salvo cuando se exprese lo contrario, "niños" será usado en sentido amplio, incluyendo tanto varones como mujeres) son generadores importantes de residuos materiales dentro de los campamentos de cazadores-recolectores y que su actividad ha sido subestimada en el análisis e interpretación de los depósitos arqueológicos (Politis, e.p.). En este artículo me propongo desarrollar esta idea y aportar elementos para la identificación de la actividad infantil en el contexto material, como un primer paso para entender la dinámica social de los cazadores-recolectores del pasado y cómo las sociedades reproducen sus repertorios culturales. Algunos de estos puntos ya fueron discutidos previamente por Lillehammer (1989) en base a una revisión de la arqueología escandinava, en un artículo pionero dentro del tema. Dentro de esta misma línea de análisis una serie de trabajos ha arribado a conclusiones similares desde perspectivas diferentes. Entre estos aportes se destaca el libro recientemente editado por Moore y Scott (1997) sobre aspectos teóricos y metodológicos y las contribuciones de Dawe (1997) y Park (1998) con originales análisis de casos de estudio arqueológicos de las llanuras norteamericanas y de los Inuit de Canadá. El resultado de estos estudios indica que los productos de la actividad infantil pueden ser reconocidos si se desarrolla una metodología apropiada. Para la construcción de esta metodología la argumentación analógica juega un papel central y las principales fuentes deben buscarse en los grupos humanos actuales, y en la información histórica y etnográfica. Este trabajo apunta a contribuir al desarrollo de instrumentos metodológicos para identificar e interpretar el agente infantil en las sociedades del pasado y se basa en una argumentación analógica usando como fuente a los indígenas Nukak.

La información obtenida entre los Nukak, un grupo cazador- recolector-pescador de la Amazonia Colombiana (Fig. 1), entre 1990 y 1996 (7 temporadas de campo, 185 días en total) permite generar expectativas materiales de la actividad infantil y evaluar bajo qué condiciones se puede esperar, en sociedades análogas del pasado, un contexto similar. Utilizando este caso de estudio etnoarqueológico como eje, los objetivos de este artículo son: a) analizar la conducta de los niños Nukak y compa-

T. P., 55, n. ${ }^{\circ} 2,1998$ 


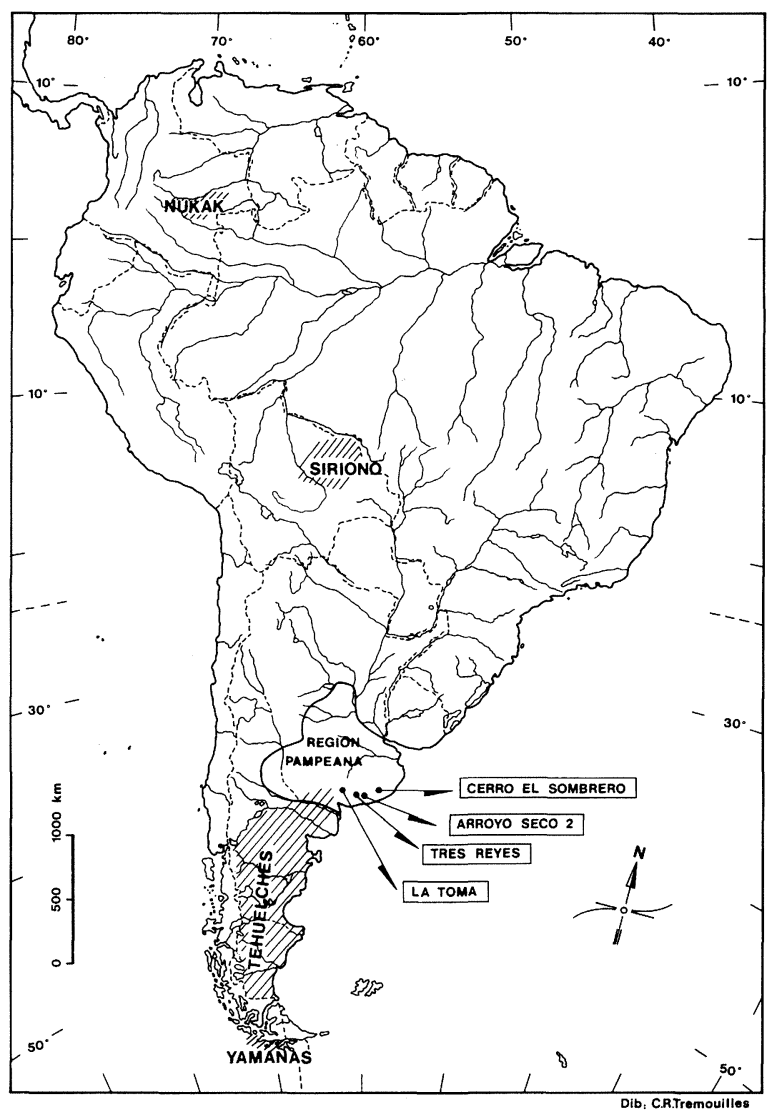

Fig. 1. Mapa de América del Sur con la localización de los grupos indígenas mencionados en el texto y la ubicación de los sitios arqueológicos de la región Pampeana de Argentina.

rarla con la de otros grupos cazadores-recolectores con el objeto de identificar algunos patrones recurrentes, b) examinar el tipo de producción material que estas conductas generan y evaluar bajo que condiciones puede que ocurran, c) confrontar estas expectativas con un caso de estudio: el registro arqueológico de algunos sitios prehistóricos de cazadores-recolectores de las llanuras pampeanas de Argentina y explorar la posibilidad de que una clase de artefactos haya sido producido por la actividad infantil.

Para los fines de este trabajo, cuando me refiero a los niños Nukak estoy incluyendo a los individuos cuyo rango de edad varía desde el tiempo que empiezan a caminar, hasta la pubertad. En términos de años esto correspondería al período entre uno a dos años y 12 a 13 años. Con anterioridad a este lapso los bebés dependen enteramente de sus padres y no generan ningún tipo de artefacto (aunque sí hay algunos que son confeccionados para ellos como por ejemplo los collares de diente de mono); en la mayoría de los casos aún no tienen nombres y se los llama genéricamente jim'bú o tóm'bú según el sexo. A partir de la pubertad, los jovenes Nukak salen de cacería y realizan todas las actividades de los adultos aunque aún no hayan completada el proceso de aprendizaje. En términos económicos, su rol es similar al de los adultos, a pesar de no pertenecer aún a ese grupo de edad y aunque socialmente todavía tengan diferencias con estos. A los 15 o 16 años un joven Nukak es económicamente tan productivo como un adulto. En el caso de las púberes ocurre lo mismo, aunque como forman pareja más temprano, el rol social que desempeñan se transforma más rapidamente, aproximándose más al de mujeres adultas (están casadas, pueden quedar embarazadas, realizan todas las tareas femeninas en el campamento, etc.).

El rango de edad que abarca la infancia parece ser variable entre las sociedades indígenas. En algunos casos es muy semejante a los Nukak, como por ejemplo entre los Guayaquí (Clastres, 1972), Yámanas (Gusinde, 1983), Sirionó (Holmberg, 1978) y Ayoréo (Bórmida, 1973). Para estos últimos la palabra disiehóde significa "niños" y abarca desde el nacimiento hasta el comienzo de la pubertad, cerca de los 12 años (Bórmida, 1973: 64). Por el contrario, entre los Hadza orientales, Hawkes et alii (1995: 689) incluyen bajo la denominación "children" a varones de hasta 17 años, una edad en la cual un joven Nukak ya es plenamente productivo y utiliza artefactos semejantes a los de los adultos. Por otro lado, Draper y Cashdan (1989) denominan "children " a niños entre 4 y 14 años.

Por último, se debe mencionar que la influencia de los niños en el registro arqueológico ha sido planteada desde hace tiempo, aunque desde una perpectiva distinta. Hammond y Hammond (1981) y Wilk y Schiffer (1979) han visto a los niños como perturbadores del registro no sólo modificando la distribución espacial de los residuos sino reinsertando en el "contexto sistémico" objetos que supuestamente habían ya pasado al "contexto arqueológico". La perspectiva planteada en este trabajo es totalmente diferente ya que en los dos artículos citados se asumía que existe un contexto previo, "normal," el producido por los adultos, que es perturbado o distorsionado por los infantes. En la presente contribución se considera a unos y a otros produciendo el registro arqueológico en un mismo nivel de jerarquía, lo que implica asumir que los niños no perturban sino que generan. 


\section{CARACTERÍSTICAS GENERALES DE LOS NUKAK}

Los Nukak son indígenas de filiación Makú (Metraux, 1948; Silverwood-Cope, 1972; Reid, 1979) que habitan la Amazonia Colombiana, en el interfluvio de los ríos Guaviare e Inirida, en el Departamento del Guaviare. En un territorio de aproximadamente $10.000 \mathrm{~km}^{2}$, viven entre $400 \mathrm{y}$ 500 Nukak que se agrupan en bandas de unos $20 \mathrm{a}$ 30 individuos (en el sector nororiental hay agrupaciones mayores que pueden llegar a 50 personas). Esta región es una zona de divisoria entre las cuencas del Orinoco y el Amazonas con cobertura vegetal típica de la foresta tropical lluviosa, aunque se observa una período seco entre diciembre y marzo. Las precipitaciones fluctúan entre $2.500 \mathrm{y}$ $3.000 \mathrm{~mm}$. anuales.

Si bien hasta 1988 había algún conocimiento sobre la existencia de indígenas de filiación Makú en el interfluvio Guaviare-Inirida (ver ReichelDolmatoff, 1967 e Informes Inéditos de laAsociación Nuevas Tribus de Colombia), es recién en ese año cuando los antropólogos y el público en general tuvieron las primeras noticias de la existencia de los Nukak (Chaves y Wirpsa, 1988). A partir de fines de la década de los 80 , los Nukak comenzaron contactos regulares con los colonos (campesinos de otras regiones de Colombia), llegando incluso hasta la capital departamental, San José del Guaviare. Desde ese momento se aceleró el proceso de aculturación y transformación de los patrones tradicionales de los Nukak (Ardila, 1992; Ardila y Politis, 1992; Cabrera et alii, 1994: 426-436; Politis, 1996b: 355-378).

A partir de los primeros contactos, se iniciaron diferentes estudios antropológicos que han esbozado las características generales de la etnía y han realizado una suerte de "etnografía de rescate" ante la velocidad de transformación de los patrones tradicionales. Entre los más importantes merecen destacarse las investigaciones sobre el territorio (Mondragón, 1991; Torres, 1994; Cárdenas y Politis, e.p.), organización social y parentesco (Cabrera et alii, 1994; Franky et alii; 1995), subsistencia (Politis y Rodríguez, 1994; Cabrera et alii, 1994; Politis, 1996b; Politis et alii, 1997; Mondragón, 1991), movilidad y asentamiento (Politis, 1992, 1996a,b), lingüística (Reina, 1990; Mondragón, 1991; Cabrera et alii, 1994) e ideología y cosmovisión (Cabrera et alii, 1994; Politis, 1996b). Asimismo se debe mencionar la copiosa información recogida por los misioneros de laAsociación Nuevas Tribus de Colombia, quienes hicieron los primeros contactos con bandas Nukak del oriente a fines de los 70 .

El ciclo anual de subsistencia ha podido ser reconstruido a partir de los trabajos citados y se ha estimado que la economía de los Nukak se basa en la caza (monos, pecaríes, aves etc.), la recolección de especies silvestres y de otros productos animales como la miel, los huevos de tortuga y el mojojoy (larvas de coleópteros del género Rhynchophorus). Hasta el presente se han identificado 123 especies vegetales utilizadas (Cárdenas y Politis, e.p.). La pesca y la horticultura en pequeña escala complementan las actividades económicas, con variada importancia según las estaciones. En forma creciente, en los últimos años, los Nukak están incorporando también alimentos que obtienen de los colonos. Sin embargo, durante el período de estudio la subsistencia tradicional se basó esencialmente en la explotación de recursos vegetales y animales no domesticados (Politis, 1996b: 58).

Los campamentos residenciales son los más frecuentes dentro del sistema de asentamiento. Durante el invierno, estos campamentos están cubiertos con hojas de platanillo y tienen una planta aproximadamente geométricá regular (triangular, cuadrangular y pentagonal); son pequeños y compactos $\left(\mathrm{n}=12, \min =32,5 \mathrm{~m}^{2}, \max =114 \mathrm{~m}^{2}, \overline{\mathrm{x}}=3,89 \mathrm{~m}^{2} /\right.$ persona). En el verano, la planta es irregular y no existe la cobertura de hojas. En esta estación los campamentos tienen dimensiones levemente superiores a los del invierno $\left(\mathrm{n}=8 ; \min =45 \mathrm{~m}^{2}\right.$, $\max =129,9 \mathrm{~m}^{2}, \bar{x}=4,46 \mathrm{~m}^{2} /$ persona). En los casos en que hay mas de una banda residiendo juntas, los asentamientos se hacen más compactos $(n=4$, $\min =99 \mathrm{~m}^{2}, \max =130 \mathrm{~m}^{2}, \overline{\mathrm{x}}=2,61 \mathrm{~m}^{2} /$ persona).

La movilidad residencial de los Nukak es muy alta y se ha estimado que la mudanza del campamento residencial se produce entre 70 y 80 veces al año. Durante nuestros trabajos de campo hemos registrado distancias entre campamentos residenciales (el abandonado y el nuevo) que varían entre 0,9 ỳ $18,1 \mathrm{~km}(\mathrm{n}=25, \bar{x}=6,25 \mathrm{~km}$, ver Tabla 3.1 en Politis 1996b).

Los Nukak están organizados en bandas autónomas, vinculadas a grupos mayores de afiliación, denominados munu, que tienen nombres definidos en referencia a su ubicación dentro del territorio. Cada banda está formada por pocas familias, usualmente no más de 5 y excepcionalmente hasta 8 . En ocasiones especiales dos bandas (o segmentos)

T. P., 55, n. ${ }^{\circ} 2,1998$ 
pueden acampar juntas por pocos días, llegando a reunir hasta 50 individuos residiendo en el mismo campamento.

\section{LA PRODUCCIÓN MATERIAL DE LOS NIÑOS}

Los niños Nukak forman una parte importante de la población de los campamentos residenciales, llegando a formar en algunos casos el $50 \%$ de los habitantes. En una muestra de 357 individuos (un alto porcentaje de la población total) se identificaron 135 menores de 10 años $(37,80 \%)$; esta cifra aumenta a 175 (49\%) si se considera los menores de 15 años (Franky et alii, 1995). Estos niños, especialmente los del primer grupo, pasan una gran parte del día dentro del campamento o en su entorno inmediato (esto es frecuente en otros grupos cazadores-recolectores, por ejemplo los !Kung, Draper, 1976). En general, los niños juegan y recorren los alrededores dentro de una distancia máxima establecida por la posibilidad de ser oídos desde el campamento. Debido a lo cerrado del bosque, los adultos pierden rápidamente el contacto visual con los infantes, por lo que, es necesario oírlos para poder ejercer alguna vigilancia.

Los niños que acompañan a sus padres en las cacerías son los que están en la etapa final de la infancia y lo mismo ocurre con las niñas cuando salen de recolección con sus madres u otras mujeres adultas del grupo. Sin embargo la participación de ambos en las salidas de adultos es un proceso progresivo desde muy pequeños. A medida que van creciendo aumenta correlativamente la frecuencia de sus salidas acompañando a los adultos, la distancia que recorren y la participación efectiva en tareas extractivas y productivas. El único punto de inflexión se podría marcar tentativamente a los 7 u 8 años, ya que a partir de esa edad se observa un acelerado proceso de preparación específica para la vida adulta. Durante este período se nota que el proceso de aprendizaje no está basado en la imitación o la enseñanza generalizada de padres a hijos, sino en una actitud de enseñanza dirigida hacia tareas específicas de cada sexo. Por ejemplo, cuando los niños tienen alrededor de 8 ó 9 años los hombres salen con sus hijos de caminata, con dardos y cerbatanas y dejan que estos prueben puntería con aves y animales pequeños. También los incentivan para que suban a las palmeras a recoger frutos e invierten algún tiempo ayudándoles a preparar cerbata- nas. Alrededor de los 10 años las niñas ya cargan con frecuencia a sus hermanos menores durante los desplazamientos y casi siempre llevan un canasto cargado con enseres. Aproximadamente a partir de esta edad empiezan a despostar los monos (una actividad exclusivamente femenina) o a colaborar en esta tarea con sus madres o niñas mayores. También practican la fabricación de pulseras de fibra ( $k d n^{\prime}$ 'yii), de cestas y de burup (canastos expeditivos de hojas. Lám. I).

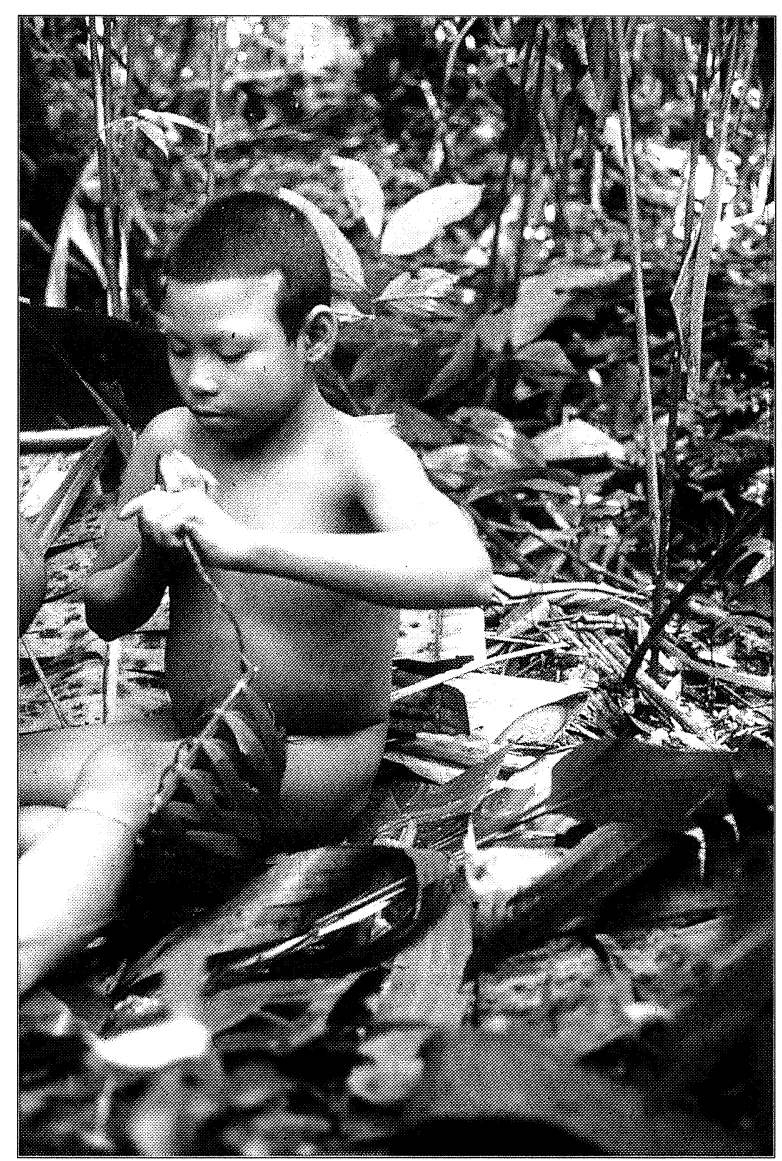

Lám. I. Niña fabricando un burup.

A pesar de que en la última parte de la infancia los niños pueden pasar una parte del día a cierta distancia del campamento residencial, el mayor tiempo transcurre dentro de éste y en su entorno inmediato. En este sentido, pasan mucho más tiempo que los adultos hombres y, según la edad, algo más que las mujeres. Los hombres salen casi diariamente a cazar, pescar, recoger frutos y a realizar una variedad de tareas extractivas hasta máximos de 11 has. y de $19 \mathrm{~km}$. de recorrido diario. Las mujeres 
pasan mucho menos tiempo fuera del campamento y sus salidas se restringen a la recolección de frutos y tubérculos en las cercanías, a la cosecha de productos vegetales cuando el campamento está próximo a un campo de cultivo o a colaborar con los hombres en las pesca. Ocasionalmente, una mujer sin hijos pequeños puede acompañar a su marido en salidas de caza y recolección más lejanas.

Cuando los niños tienen menos de 2 años, siempre están cerca de las madres y éstas los llevan en las salidas afuera del campamento para recolectar, cosechar o pescar. Después de los 2 ó 3 años y en la primera parte de la infancia pueden quedarse en el campamento bajo la vigilancia de niños mayores o púberes o acompañar a sus madres. Los hermanos mayores juegan un rol importante en el cuidado de los más pequeños y pasan gran parte del tiempo a cargo de ellos. Esto es común en otras sociedades tradicionales (peer rearing, Weisner y Gallimore, 1977). En algunos casos, hay una o dos horas al día en que el campamento residencial queda ocupado sólo por un grupo de infantes cuyas edades varían entre 3 y 11 años. A partir de los 6 ó 7 años, los niños pueden hacer algunos recorridos cortos cercanos al campamento, acompañados por niños mayores, durante los cuales recolectan algunos frutos accesibles, pescan y recogen cangrejos en charcas y arroyos de los alrededores o, simplemente, juegan y se divierten.

A pesar de estas salidas, sólos o con sus madres, los niños pasan la mayor parte de las $12 \mathrm{~h}$. diurnas, $\mathrm{y}$ todas las nocturas, dentro o en las inmediaciones del campamento residencial. En este lugar, usan y, eventualmente fabrican, tres clases de juguetes:

Clase 1: Artefactos exclusivamente confeccionados para jugar que tienen un diseño específico.

Clase 2: Artefactos que copian la forma de los de adultos pero que tienen un tamaño menor y que son usados en funciones similares a los de adulto o con fines lúdicos.

Clase 3: Artefactos de los adultos, enteros o fragmentados, que son utilizados con fines lúdicos.

Dentro de la clase 1 se encuentran los siguientes juguetes: los aros de bejucos, los trompos de frutos, columpios de corteza o fibras y los cantos rodados. Estos no tienen artefactos homólogos entre los adultos y son fabricados por los mismos niños (a veces con la ayuda de sus padres) con modificaciones menores o incluso (como los cantos rodados) sin ningún proceso de formatización. Pueden incluir juguetes muy pequeños, como los trompos de frutos (Lám. II), hasta otros de tamaño mediano

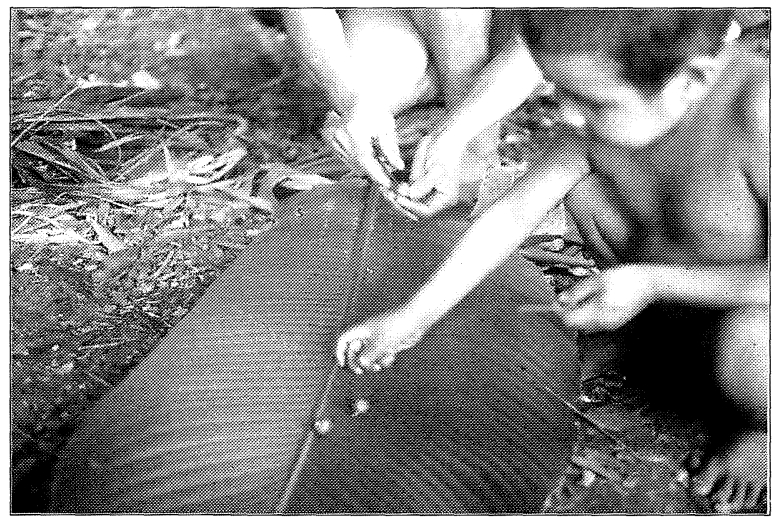

Lám. II. Niño jugando con trompos de frutos en el campamento residencial. La madre le está ayudando a armar los trompos con una espina.

como los aros de bejuco de aproximadamente un metro de diámetro. Dentro de este grupo es interesante mencionar los guijarros que en algunas oportunidades los niños Nukak traen desde el "Cerro de las Cerbatanas" cuando la banda visita estas sierras para recolectar cañas para cerbatanas (Politis, 1996b: 284). Estas rocas, que sacan del lecho de los arroyos que bajan de la formación rocosa son trasladados de un lugar a otro durante semanas o meses, hasta que finalmente se van abandonando o perdiendo en los campamentos o en sus alrededores. Los guijarros son usados exclusivamente por los niños para jugar y no se ha observado ninguna otra función.

La clase 2 contiene muchos más juguetes e incluye prácticamente todos los instrumentos hechos por adultos, pero a escala menor. Entre éstos se destacan: cestas, cerbatanas, dardos, arcos, flechas, balayes (cestas planas de trama más abierta), burup, totumos (recipientes de calabaza, de varias especies), arcos, vasijas de alfarería y lanzas. En esta segunda clase hay que hacer una distinción importante. Por un lado algunos artefactos son hechos por adultos, en tamaño más pequeño, para que sean utilizados por los niños, cumpliendo una función similar a la de los mayores. La única diferencia entre los artefactos de los adultos y de los niños son las dimensiones, que se adecúan a la edad y el tamaño del niño, pero la calidad de confección y la función son las mismas. Dentro de este subgrupo se encuentran los totumos y los recipientes de alfarería. Por otro lado están las réplicas de los instrumentos de los mayores, hechos por los mismos niños o sus padres, para jugar o practicar. Estos no son usados con la misma función que les dan los adultos, 
aunque a veces se aproxima, y la calidad de confección es menor. Esta baja calidad se debe a dos razones: a) cuando los hacen los adultos, la tecnología tiene un caracter expeditivo, debido al fin lúdico de los artefactos y no los confeccionan con el mismo esmero; b) cuando los hacen los niños, la baja calidad se debe a sus limitaciones técnicas.

Dentro de este sub-grupo se encuentran cestas, balayes, cerbatanas, dardos, arcos y lanzas. Es frecuente ver a niños de 5 a 10 años de edad, disparando con pequeñas cerbatanas, apuntándole a cualquier objeto o confeccionando los dardos. Lo mismo sucede con los arcos y flechas que usan para arrojar al tronco de árboles o a cualquier otro blanco potencial (Lám. III). Asimismo, las niñas fabrican cestas, balayes, vasijitas de cerámica y totumos que luego usan para poner diversos objetos y jugar con ellas.

El tamaño de estos objetos está en relación al del niño y virtualmente, dentro de un rango determina-

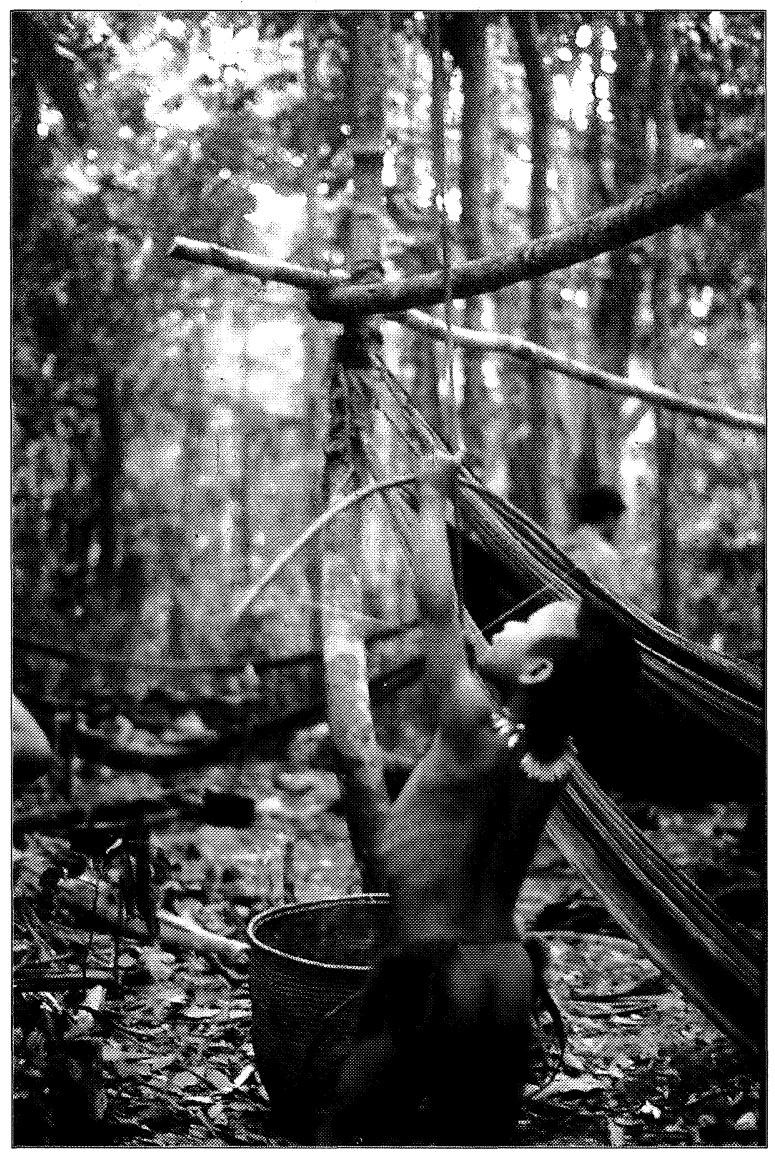

Lám. III. Niño jugando con un arco y flecha en un campamento de verano. do, pueden existir todas las medidas. En el caso de las cerbatanas, por ejemplo, la más pequeña que nosotros registramos era de $0,82 \mathrm{~m}$. de longitud. De allí en adelante hay una gradación hasta las de los adultos que llegan a los 3,20 m. La diferencia entre la de los adultos y la de los niños se dá por la relación que existe entre el largo de la cerbatana y la estatura y habilidad del usuario. En los niños, la longitud de la cerbatana es en general menor que su estatura. En los púberes ambas medidas se aproximan (entre 1,40 y 1,60 m. aprox.) y cuando llegan a adultos y a su estatura definitiva y van adquiriendo pericia en el manejo de este arma, la longitud llega a valores cercanos a los $3 \mathrm{~m}$. Finalmente, las cerbatanas que se hacen más largas, también se confeccionan mejor pues se van acercando a su función específica para la caza y van perdiendo su caracter lúdico y pedagógico.

La clase 3 está formada por artefactos de adultos, enteros o rotos, que son usados por los niños como juguetes de manera circunstancial. Estos objetos no son modificados y la actividad de los infantes afecta sólo a su distribución espacial. Esta clase es más utilizada por los más pequeños, que toman como juguete cualquier objeto que tengan cerca.

Los artefactos de los niños se diferencian de los de los mayores también por el lugar de descarte. En el primer caso, los juguetes son desechados dentro del campamento o en su entorno inmediato porque allí es donde llevan a cabo la mayoría de las actividades lúdicas. Como consecuencia de esto quedan abandonados en el piso de los campamentos pequeños dardos y flechas (generalmente enteros), dispersos entre las unidades domésticas o en los depósitos secundarios de basura (pilas de desechos que se acumulan a los costados de las entradas/salidas de los campamentos de la estación lluviosa, Politis, e.p.). Estos objetos obviamente no tienen relación con ningún evento de caza o pesca.Además, como en general estos juguetes son esencialmente expeditivos, se descartan en el campamento residencial cuando éste se abandona y raramente son transportados de un sitio a otro. Este es también el caso de las cerbatanas y los arcos. Hay algunas excepciones, como por ejemplo las cestas, los balayes y los guijarros que se llevan de un campamento a otro hasta que por ruptura, extravío o por haber dejado de ser motivo de interés, terminan quedando en alguno de los asentamientos (pero no en el que se fabricaron).

Otro ejemplo interesante lo constituyen los pequeños campamentos que los niños construyen en 
los alrededores de los campamentos residenciales, y en donde pasan varias horas jugando e imitando las actividades de los adultos. En estos pequeños asentamientos se encuentran algunos postes y travesaños en donde cuelgan las hamacas (que a la noche llevan de nuevo al campamento mayor) y hay algunos fogones. En estos lugares quedan en el piso, además de restos de comida, toda suerte de objetos de las tres clases, que fueron utilizados como juguetes.

A veces los niños también construyen como divertimento, pequeñas viviendas dentro del mismo campamento residencial. Estas son una réplica en escala menor de una vivienda de invierno: dos postes, un travesaño central y una hilera de hojas pequeñas de platanillo como techo (Lám. IV).

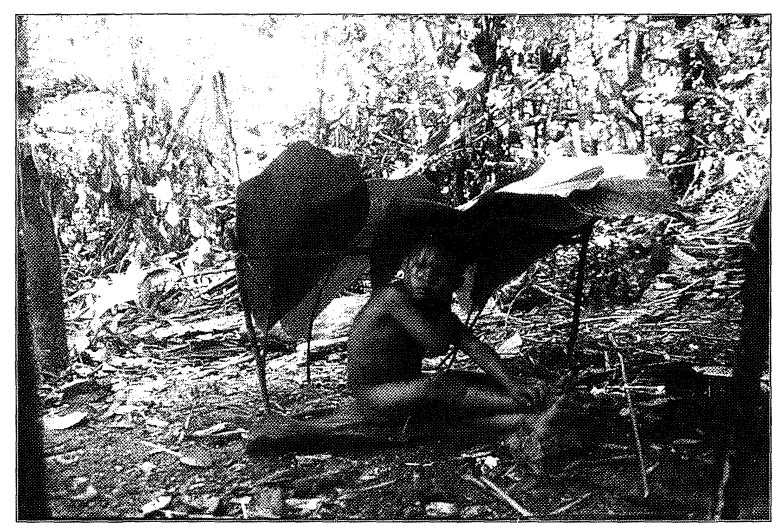

Lám. IV. Pequeña réplica de una vivienda de invierno, construida en un borde de un campamento residencial.

Es posible generar expectativas arqueológicas para reconocer por lo menos dos de las tres clases de artefactos infantiles dentro del registro arqueológico. En principio, la clase 3 es indiferenciable, en cuanto a morfología, tecnología y dimensiones, pero las otras dos tienen características que permiten su identificación dentro de un contexto arqueológico (Tab. 1).

\begin{tabular}{|c|c|c|c|}
\hline & Homólogos & Tamaño & Tecnología \\
\hline CLASE 1 & no & $\begin{array}{c}\text { variable } \\
\text { (mediano o } \\
\text { pequeño) }\end{array}$ & $\begin{array}{c}\text { baja calidad o } \\
\text { sin modifica- } \\
\text { ción }\end{array}$ \\
\hline CLASE 2 & sí & más pequeños & menor calidad \\
\hline
\end{tabular}

Tab. 1. Diferencias de los artefactos de niños con respecto a los de adultos
La otra forma de identificación, que debe ser analizada en conjunto con las características precedentes, es el lugar de descarte. Mientras que los artefactos de la clase 1 son descartados en el lugar donde fueron usados para actividades lúdicas, en la clase 2 los objetos son desechados donde fueron usados como juguetes, que en muchos casos son diferentes al lugar donde se abandonan sus homólogos adultos. Esto es notable en el caso de los dardos, las flechas las lanzas y otros objetos de hombres adultos que son abandonados (usualmente rotos) en sitios afuera y a cierta distancia de los campamentos residenciales, donde se llevó a cabo la cacería. En artefactos tales como vasijas, cestas, balayes etc., tantos los de adultos como los de niños son descartados en el campamento residencial. Obviamente en todos los casos, el lugar de descarte inicial puede cambiar debido a las actividades de limpieza del campamento (Politis, e.p.).

En el análisis propuesto es necesario poder discriminar entre el diseño pequeño y la disminución de tamaño debido al uso. En general, esta disminución está acompañada de otros rasgos que permiten identificar el uso intenso (por ejemplo filos muy abruptos en los artefactos líticos) y no se dá proporcionalmente en toda la pieza sino sólo en una dimensión. En las cerbatanas por ejemplo es el largo, en los cuchillos líticos es el ancho, en los instrumentos pasivos de molienda es el espesor. También es posible identificar entre artefactos de infantes y miniaturas como se ha planteado para los Inuit (Park, 1998). Esta distinción es posible porque en el caso de las miniaturas Inuit que usan los shamanes y las que se entierran como ajuar funerario, la calidad de confección es muy alta, una característica que no tienen la mayoría de los artefactos infantiles.

Por último, en todos estos casos la distinción también debe basarse en el contexto de depositación, que es diferente cuando se trata de artefactos infantiles que cuando son miniaturas, instrumentos pequeños o muy usados.

\section{UN CASO DE ESTUDIO: CONFRONTACIÓN CON EL REGISTRO ARQUEOLÓGICO PAMPEANO}

Los materiales recuperados en varios sitios arqueológicos prehistóricos de cazadores-recolectores de la Región Pampeana de Argentina son susceptibles de ser analizados usando como referencia 
las expectativas generadas a partir de la información de los Nukak y de datos etnográficos de otros cazadores-recolectores (por ej. Tehuelches de la Patagonia, Yámanas de los canales Fueginos del extremo surAmericano, Sirionó del oriente boliviano, varios grupos de las Planicies Norteamericanas, etc.).

La presencia infantil ha sido identificada en muy pocos casos en la arqueología pampeana. El caso más obvio han sido los esqueletos de infantes recuperados en varios sitios, especialmente en Arroyo Seco 2, en el grupo de entierros datados en ca. 6400 años A.P. (Politis, 1989; Barrientos, 1997). La otra evidencia proviene del sitio Monte Hermoso 1, en donde se han identificado numerosas pisadas y rastros asignados a niños de diversa edad (Bayón y Politis, 1997).

Con excepción de estos dos casos, el resto del material arqueológico fue asumido como generado por adultos y en ningún caso se ha planteado o discutido la posibilidad de que alguno de las decenas de miles de artefactos y desechos hallados en los 290 sitios registrados hasta el presente en la Región Pampeana (ver inventarios en Berón y Curtoni, 1995 y Barrientos et alii, 1996) hallan sido objetos de o para infantes. Sin embargo, en algunos sitios hay piezas que podrían corresponder a las dos clases de artefactos definidas previamente.

\section{Bolas de boleadora}

Dentro de los 41 esqueletos humanos hallados en el sitio a cielo abierto, multicomponente deArroyo Seco 2 (Politis, 1984; Fidalgo et alii, 1986; Politis, 1989; Barrientos, 1997) se encuentran 9 que corresponden a infantes. La mayoría de estos esqueletos contiene ocre rojo en el sedimento que rodea a los huesos y cuentas de colmillos de cánidos, a modo de collares y pulseras, alrededor del cuello, las muñecas y los tobillos. En un caso, el enterramiento Nro. 4 (esqueleto nro. 9, asignado a un niño de pocos años) de una antigüedad estimada en $c a$. 6400 años A.P., se recuperó además como ajuar funerario cerca del cráneo una pequeña bola de basalto negro (AS2/34/11) de $26 \mathrm{~mm}$. de diámetro y 28 gr. de peso (Lám.V). Esta bola es similar a la de las típicas boleadoras pampeanas sin surco pero tiene dos particularidades. Una es que está confeccionada en basalto, una materia prima raramente utilizada para este artefacto. La otra es su tamaño, sensiblemente menor al de las bolas que se hallan en abundancia en la Región Pampeana $(n=126$,

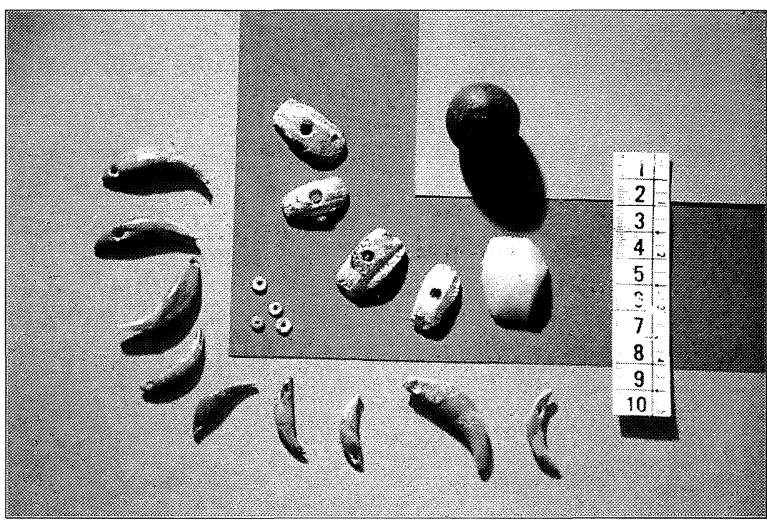

Lám. V. Ajuar funerario del enterramiento nro. 4 de Arroyo Seco 2. Se destaca la pequeña bola de basalto negro (arriba a la derecha).

diam. $x=63 \mathrm{~mm}$., peso $\mathrm{x}=372,5$ gr., en base a los datos de González, 1953).

Entre los Tehuelches de la Patagonia hay varias referencias de Musters (1997) a fines del siglo pasado que señalan que los niños jugaban con bolas de boleadora de menor tamaño que la de los adultos y que las ataban con tientos de ñandú. También se relata un accidente producido por una boleadora durante los juegos infantiles.

Se propone que la boleadora del enterramiento 10 de Arroyo Seco 2 corresponde a una artefacto infantil, que se incluye dentro de la clase 2 y que fue confeccionada por adultos. Las evidencias para sustentar esta hipótesis son: a) tiene un artefacto homólogo de adulto; b) es de tamaño menor; c) se encuentra en el contexto funerario de un niño; y d) hay datos etnográficos regionales que indican el uso de boleadoras pequeñas como juguetes. La buena manufactura del artefacto no encuadra totalmente con las expectativas generadas a partir del caso Nukak. Esto se explicaría parcialmente por el tipo de materia prima ya que el basalto tiene superficies pulidas naturalmente, por lo cual la confección de una pequeña boleadora sólo requeriría redondear algún guijarro ovoide. La fineza del acabado no sería significativa como indicador de una alta calidad de manufactura, pues, como se ha expresado, la superficie ya tiene un pulido natural.

\section{Rodados costeros}

En varios sitios de la Región Pampeana se han hallado rodados costeros sin ninguna modificación, especialmente en componentes del Holoceno Me- 
dio (ca. 7000 a 3500 años A.P.) y Tardío ( $c a$. después del 3500 A.P.): Zanjón Seco 2, Componente Superior de La Toma y componentes Medio y Superior deArroyo Seco 2. Se han registrado también en otros sitios pampeanos sin asignación temporal.

Estos rodados son de tamaño mediano o pequeño y se encuentran naturalmente en el litoral pampeano, disminuyendo de tamaño del suroeste al noreste. En general se hallan en la playa, cuando hay marea baja y forman bancos en los sectores rocosos. Los artefactos confeccionados sobre rodados, básicamente mediante la técnica bipolar, son muy abundantes en la faja medanosa litoral, en donde se encuentran abundantemente en superficie entre las dunas (de Aparicio, 1932; Austral, 1965; Politis, 1984). En base a estos materiales se ha planteado la existencia de grupos adaptados a la explotación de los recursos marinos (Bórmida, 1969). En los sitios del interior de la Región Pampeana, los artefactos sobre rodado costeros son ocasionales (Politis, 1984) y en algunos casos han sido reducidos por técnica bipolar.

Los rodados del sitio Arroyo Seco 2 son relativamente abundantes en los niveles superiores y son de tamaños mediano a pequeños (Tab. 2) (Lám.VI); no presentan ninguna modificación macro ni microscópica que indique uso (Leipus, 1997). El caracter antrópico de la presencia de estos cantos en el sitio está dado por su situación contextual y por la lejanía $(50 \mathrm{~km}$.) a la costa marina, en donde se encuentran naturalmente los rodados (Fig. 1).

Teniendo en cuenta que los rodados fueron transportados al sitio con algún objetivo, se plantea que fueron usados para actividades lúdicas por los niños y que se pueden incluir dentro de la clase 1. Las evidencias que sustentan esta hipótesis son: a) no tienen ninguna modificación o trabajo; b) no se observan evidencias de uso para algún tipo de actividad tecnológica tal como percutir, presionar, alisar o pulir; c) la baja calidad de la materia prima, que no justificaría el transporte de estos nódulos pequeños y medianos. La existencia de análogos

\begin{tabular}{|l|l|c|c|c|}
\hline \multicolumn{1}{|c|}{ Nro. } & Unidad Estrat. & $\begin{array}{c}\text { Long. } \\
\text { en cms. }\end{array}$ & $\begin{array}{c}\text { Ancho } \\
\text { en cms }\end{array}$ & $\begin{array}{c}\text { Peso } \\
\text { en gr. }\end{array}$ \\
\hline 50.3 & Parte sup. de Y & 6,20 & 2,86 & 53,2 \\
33.XI.1 & Y & 4,34 & 2,99 & 42,9 \\
A36.L & X trans. con Y & 3,75 & 2,65 & 23,1 \\
A39.13 & Parte sup. de Y & 6,14 & 3,29 & 77,7 \\
50.6 & Parte sup. de Y & 5,79 & 5,29 & 71,1 \\
64.II.2 & Y & 4,85 & 3,81 & 61,4 \\
S.r. & indet. & 5,69 & 4,13 & 85,7 \\
\hline
\end{tabular}

Tab. 2. Rodados costeros de Arroyo Seco 2.

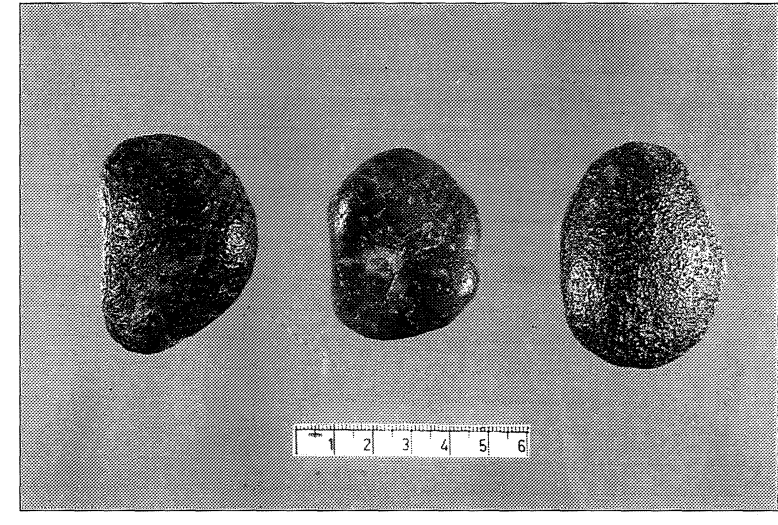

Lám. VI. Cantos rodados del sitio Arroyo Seco 2.

etnográficos (los Nukak), que trasladan y abandonan en los campamentos residenciales guijarros sin modificar cuya función es exclusivamente lúdica infantil, indica que este tipo de conductas se registra entre cazadores-recolectores. Esto apoyaría, aunque no probaría, la hipótesis enunciada.

La alternativa de que los nódulos sean para usar como materia prima de instrumentos en el sitio es poco probables debido, no sólo a la calidad de la materia prima y a las limitaciones que impone el tamaño y la forma redondeada, sino al hecho de que no se han encontrado en el sitio núcleos agotados de esta roca ni desechos de las primeras etapas de reducción.

\section{Puntas de proyectil}

Existen muy pocas evidencias etnográficas de la región Pampeana de Argentina que nos permitan derivar expectativas arqueológicas para el uso de las puntas de proyectil, pero la información con respecto a otros grupos cazadores-recolectores es relativamente abundante. Hay, por ejemplo, datos interesantes de Gusinde (1983) sobre los Yámanas, en donde relata que la manufactura de los instrumentos, inclusive las puntas de flecha, comenzaba a ser enseñada por los padres a sus hijos desde la edad de 3 años en adelante. Una de estas citas es muy clara al respecto: "Un adulto, generalmente el padre, les construye [a los hijos] reproducciones de las armas y utensilios de que se sirven los hombres para cazar... Naturalmente estas piezas se arruinan pronto o se pierden; pero infatigablemnte los adultos construyen un sustituto y cuanto más crece el niño, más grandes son tales utensilios" (Gusinde, 1983: 729). Entre los Sirionó, Holmberg (1978: 185) relata que

T. P., 55, n. ${ }^{\circ} 2,1998$ 
antes de que un niño varón haya cumplido los 3 años, el padre ya le ha hecho un arco y flechas en miniatura, aunque de manera simbólica pues aún no los puede usar. Luego de esa edad, el infante comienza a practicar con algún tipo de arco y pasa muchas horas disparando a blancos inertes para divertirse y practicar; para los 8 años ya ha cazado algún animal pequeño y luego de esa edad comienza a acompañar a su padre en las cacerías. Holmberg también menciona que las flechas de los niños siempre tienen las puntas rotas y sus arcos disparan con muy poca fuerza. Un trabajo de Dawe (1997) ha recopilado abundantes citas históricas y ha demostrado como entre diversos grupos de las llanuras norteamericanas la confección de puntas de proyectil pequeñas, para que los niños jugaran y adquirieran las habilidades para la caza, era muy frecuente.

Todos los ejmplos mencionados coinciden con lo observado entre los Nukak, en cuanto al tamaño menor del equipo y a la existencia de homólogos entre los adultos. Hay también similitud, referida a que las puntas eran hechas para los niños por los adultos (como algunos artefactos nukak) al principio de la infancia y luego por los mismos infantes, y también en que había una proporción entre el tamaño del artefacto y el del niño. En este sentido, se ha observado que las flechas y los arcos eran construidos dependiendo de la altura del arquero (Bourke, 1891) y por lo tanto en el registro arqueológico es esperable encontrar uncontinuum de tamaños de puntas de proyectil determinado, entre otros factores, por la altura del usuario (Dawe, 1997). En suma, estas observaciones permiten derivar expectativas arqueológicas similares a las basadas en los Nukak, con respecto a los instrumentos de la clase 2 , en sus dos variantes (confeccionado por los mismos niños o por los padres).Tomando estas expectativas en consideración, se analizará el material de algunos sitios pampeanos con puntas de proyectil pequeñas: Cerro El Sombrero (Cima), LaToma y LagunaTres Reyes.

En el sector suroriental del sistema de Tandilia, Flegenheimer $(1991,1995)$ ha estudiado un grupo de sitios con componentes ubicados en el Pleistoceno Final, que han sido asignados a un mismo sistema cultural: Cerro La China 1, 2, y 3, Cerro El Sombrero Cima y Cerro El Sombrero Alero. Estos componentes han sido consistentemente datados entre 10.000 y 11.000 años A.P. (Flegenheimer y Zárate, 1997). Uno de estos sitios, Cerro El Sombrero Cima, fue interpretado como un sitio de actividades específicas en donde se habría producido la confección de puntas de proyectil del tipo "cola de

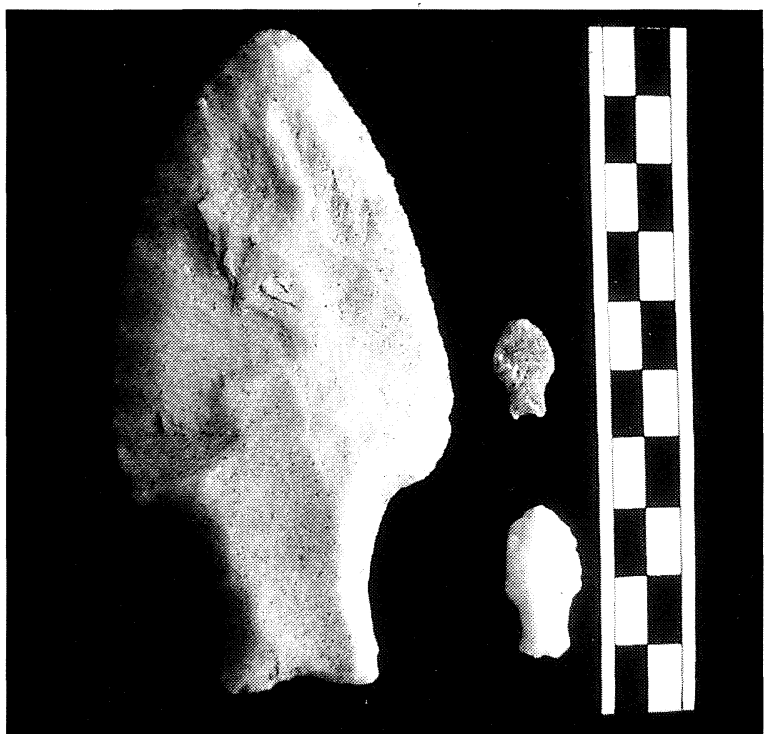

Lám. VII. Puntas de proyectil «cola de pescado» del sitio Cerro El Sombrero Cima. El ejemplar grande es el S12.105.1. El más pequeño corresponde a la pieza S12.301.1. Foto cortesía de Nora Flegenheimer.

pescado" (también llamadas Fell 1 o Fell's Cave Stemmed) y el reemplazo de puntas rotas de los astiles (con lo cual se explicaría la gran cantidad de pedúnculos que se registran en el sitio). En superficie y en estratigrafía se han hallado 67 puntas y pedúnculos de "cola de pescado", de variado tamaño. Las más grandes llegan hasta $95 \mathrm{~mm}$. (pieza S12.105.1, Lám.VII) siendo de tamaño mayor que el promedio de este tipo de puntas en el Cono Sur (Politis, 1991). Entre las de menor tamaño, se destacan algunas muy pequeñas (diam. max. $<30 \mathrm{~mm}$.), con una calidad de manufactura notablemente inferior a las medianas y grandes. El caso más claro es el ejemplar S12.301.1 (aprox. $17 \mathrm{~mm}$. de largo $\times 10$ $\mathrm{mm}$. de ancho; Fig.1.c en Flegenheimer y Zárate, 1989 y Lám. VII).

Se propone que estas puntas "cola de pescado" pequeñas han sido realizadas por niños, probablemente en la última parte de su infancia, cuando estaban en un proceso de aprendizaje tecnológico por imitación de las actividades de sus padres. Estos artefactos se incluirían en la clase 2. Esta hipótesis se basa en las siguientes evidencias: a) artefactos homólogos en el mismo contexto b) tamaño pequeño c) baja calidad tecnológica.

En diversos componentes del Holoceno Tardío se han recuperado puntas de proyectil bifaciales triangulares pequeñas apedunculadas. Estas puntas pequeñas están confeccionadas en cuarcita, calce- 
donia y chert y se caracterizan por la alta calidad y precisión de la técnica de fabricación. En general, estas puntas tienen una muy baja frecuencia de registro en los sitios, con la única excepción de la Localidad Lobería 1 (Ceresole y Slavsky, 1985).

El componente superior del sitio La Toma ha sido considerado como un lugar de actividades múltiples datado en ca. 1.000 años A.P. (Madrid y Politis, 1991). En este componente se han recuperado 3 puntas de proyectil, dos fragmentadas y una tercera casi completa. Una de estas, el ejemplar LT.2/3.XVI.2 es triangular de cuarzo ( $11 \mathrm{~mm}$. x 15 $\mathrm{mm}$. x $2 \mathrm{~mm}$.), está casi completa y sólo tiene el ápice fracturado. La técnica de manufactura es de menor calidad que las otras dos y tiene además los lados asimétricos.

En el Componente Superior de Laguna Tres Reyes, datado entre ca. 2200 y 1800 añosA.P. (Madrid et alii, 1991; Madrid y Salemme, 1991), se han hallado 9 puntas de proyectil, 3 de la cuales comparten las características de la pieza LT.2/3.XVI.2. Se trata de ejemplares muy pequeños, de mala confección y asimétricos. Uno, la pieza TR1.6.III.19a $(13 \times 12 \times 2 \mathrm{~mm}$.), es de cuarcita y tiene el ápice frac-

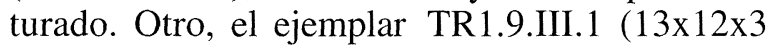
$\mathrm{mm}$.), está completo y es también de cuarcita. La

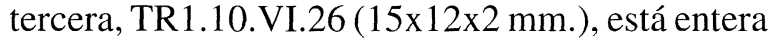
$\mathrm{y}$ es de calcedonia.

Se propone que la punta de proyectil LT.2/3. XVI.2 y las 3 del Componente Superior de Laguna Tres Reyes son instrumentos confeccionados y usados por niños. Esta hipótesis se basa en: a) tienen homólogos dentro del mismo contexto; b) son de tamaño pequeño, ubicado en el límite inferior del rango de variación de las puntas triangulares pequeñas del Holoceno Tardío de la región Pampeana; c) son de baja calidad técnica; d) son asimétricas lo que las hace aerodinámicamente ineficaces y por lo tanto inadecuadas para punta de un arma arrojadiza como una flecha.

\section{CONSIDERACIONES FINALES}

En este trabajo he presentado uno de los aspectos casi inexplorado en la arqueología que es la producción material de los niños y he examinado su aporte en la formación de los sitios arqueológicos de la región Pampeana. Siguiendo la preocupación de otros autores (Lillehammer, 1989) he tratado de aportar una metodología para identificar la participación infantil en el registro material, como un paso inicial para discutir su agencia en las sociedades del pasado. El hecho de que los infantes formen un porcentaje importante de las poblaciones de cazadores-recolectores y que pasen la mayor parte del tiempo en o alrededor de los campamentos residenciales tiene dos implicaciones relevantes para la arqueología. La primera es que debemos esperar una cantidad significativa de materiales producidos por o para los niños en determinado tipo de sitio. La segunda es que la identificación de conjuntos infantiles diversos (especialmente correspondientes a la infancia temprana) sería un buen indicador de la función del sitio ya que la presencia de estos objetos sugiere al menos una proximidad al campamento residencial.

Teniendo en cuenta el primer enunciado es fácil imaginar la cantidad de desechos y artefactos líticos que los niños de una banda pueden producir dentro del campamento. Desechos y artefactos que no tienen ningún objetivo económico o utilitario (strictu sensu) sino que han sido generados para aprender y entretenerse. Estos elementos no siguieron la trayectoria esperada dentro de un modelo de eficiencia de utilización de la materia prima para una tecnología dada ni tampoco están en directa vinculación con la producción de los artefactos necesarios para la obtención y procesamiento de ciertos recursos. En base a la información etnográfica de cazadores-recolectores, se podría anticipar que una parte significativa de los contextos líticos de los sitios arqueológicos asignados a campamentos residenciales ha sido generada por los niños. Esto puede incluir desde la práctica en la reducción de nódulos y núcleos, hasta el entretenimiento con lascas y desechos diversos descartados por los talladores adultos. Se abre así una gama de posibilidades para interpretar la cultura material, cuando consideramos que no todo es consecuencia de una actividad consciente, dirigida y planeada hacia la obtención de los artefactos "utilitarios", sino que por el contrario, el registro arqueológico es el efecto de una multiplicidad de causas que incluyen la enseñanza y aprendizaje, el juego y la diversión, la búsqueda de prestigio y el mantenimiento de estatus.

Los niños, como así también los ancianos, hacen las cosas de modo diferente a los adultos. Los primeros tienen como objetivo aprender y entretenerse, los segundos enseñar y mantener el prestigio. Ambos grupos de edad contribuyen significativamente a generar el registro arqueológico, especialmente en campamentos residenciales. ¿Qué porcentaje de los desechos que llevan a reconstruir cadenas 
operativas ha sido generado en un proceso lineal de producción de un equipo de artefactos utilitarios?, ¿cuánto ha derivado de la acción recurrente y constante de los niños jugando y practicando dentro de los campamentos? Esto, salvo pocas excepciones (por ejemplo Bodú et alii, 1990; Nami, 1994) no ha sido planteado en el estudio de la tecnología lítica de los cazadores-recolectores del pasado. Sin duda, la respuesta es difícil y distinta para caso de estudio, pero en principio la mejor forma de abordar el análisis sería desde una perspectiva que diera cabida a múltiples agentes, no todos tratando de usar la materia prima de manera óptima, no todos suficientemente entrenados y habilidosos, no todos preocupados por obtener artefactos útiles y eficientes en términos tecno-económicos.

El rango de variación de tamaño y calidad de algunos artefactos (especialmente las armas de caza) según la edad y la estatura del usuario tiene también fuertes implicaciones para el análisis arqueológico. En primer término indica que los artefactos de adultos y los de niños no se separan en dos conjuntos discretos sino que se deben visualizar como un continuum de tamaño y calidad. En un extremo se encontrarán los instrumentos mas pequeños y posiblemente peor hechos (como por ejemplo las puntas "cola de pescado" más pequenas del Cerro El Sombrero Cima), en el otro los más grandes y más elaborados. En el medio, infinitas combinaciones dependiendo, entre otros factores, de la estatura, la edad y la habilidad del fabricante o usuario. En consecuencia, algunos artefactos usados frecuentemente como diagnósticos en la interpretación arqueológica, pueden variar en la forma y las dimensiones en función de estas tres propiedades. La variabilidad en el diseño de las puntas de proyectil, por ejemplo, ha sido ampliamente utilizada como indicador de distinto tipo en la arqueología de los cazadores-recolectores (por ejemplo etnicidad, idiosincracia, habilidad tecnológica, jerarquía social, función, etc.). Por supuesto, no niego que estas u otras sean causas de la variación estilística y técnica de las puntas de proyectil u otros artefactos (la literatura etnográfica abunda en ejemplos al respecto, i.e. Wiessner, 1983), pero propongo que la variabilidad artefactual también es consecuencia del grupo de edad y que esto debe ser considerado en el análisis del registro arqueológico.

Las expectativas arqueológicas generadas a partir de la información etnoarqueológica, etnohistórica y etnográfica no deben ser tomadas como recetas de aplicación mecánica y universal sino como guías o referencias para comenzar a explorar la cultural material de los niños en paisajes arqueológicos específicos y a partir de allí discutir la agencia infantil en las sociedades del pasado. En este sentido, el fin de este trabajo es contribuir a entender los contextos arqueológicos bajo estudio no sólo como una producción de una población de adultos, sino como el resultado de la actividad de actores sociales diversos en un escenario de producción y reproducción cultural.

\section{AGRADECIMIENTOS}

Los trabajos de campo entre 1991 y 1994 fueron realizados gracias a dos subsidios, nros. 5389 y 5704, de laWenner-Gren Foundation forAnthropological Research. Desde 1995 en adelante el proyecto fue radicado en el InstitutoAmazónico de Investigaciones Científicas SINCHI (Colombia) y financiado con fondos de esta institución. Durante mis estadías entre los Nukak me acompañaron alternativamente Julián Rodríguez, Dairon Cárdenas y Gustavo Martínez. Revisaron y comentaron las primeras versiones de este artículo: Almudena Hernando, Patricia Madrid y Hugo Nami. Nora Flegenheimer aportó la lámina VII. Agradezco a todas estas personas e instituciones, y especialmente a los Nukak, sin cuya ayuda este trabajo no habría sido posible. Los errores y omisiones que contiene este artículo son, por supuesto, de mi exclusiva responsabilidad.

\section{BIBLIOGRAFÍA}

Aguilar, M. (1994): "Portraying Society through Children. Play among the Waso Boorana of Kenya". Anthropos, 89: $29-38$.

Amorós, C. (1997): Tiempo de feminismo. Sobre feminismo, proyecto ilustrado y postmodernidad. Ed. Cátedra. Universidad de Valencia. Instituto de la Mujer. Madrid.

Ardila, G. (1992): "Los Nukak-Makú del Guaviare: mi primer encuentro con la gente de las palmas (etnografía para la arqueología del poblamiento de América)". América Negra, 3: 171-189.

Ardila, G. y Politis, G. (1992): "La situación actual de los Nukak de laAmazonía Colombiana: Problemas y Perspectivas". Revista de la Universidad Nacional de Colombia, 26: 2-6.

Austral, A. (1965): "Investigaciones prehistóricas en el curso inferior del río Sauce Grande (Pdo. de Coronel de Marina Leonardo Rosales)". Trabajos de Prehistoria, XIX. Madrid. 
BAKER, M. (1997): "Invisibility as a sympton of gender categories in archaeology". En J. Moore y E. Scott (eds.): Invisible People and Processes. Writing Gender and Childhood into European Archaeology. Leicester University Press. Londres: 183-191.

BARRIENTOS, G. (1997): Nutrición y dieta en las poblaciones aborígenes prehispánicas del sudeste de la Región Pmapeana. Tesis Doctoral. Facultad de Ciencias Naturales y Museo. Universidad Nacional de La Plata. La Plata, Argentina.

BAYÓn, C. y Politis, G. (1997): "Estado actual de las investigaciones en el sitio Monte Hermoso 1 (Pcia. de Buenos Aires)". Arqueología, 6: 83-115. Facultad de Filosofía y Letras. Buenos Aires.

Berón, M. y Curtoni, R. (1995). Atlas arqueológico de la Provincia de La Pampa. Informe Consejo Federal de Inversiones y Subsecretaría de Cultura de la Pcia. de La Pampa.

Bodú, P.; Karlin, C. y Ploux, S. (1990): “Who's who? The Magdalenian flintknappers of Pincevent". En E. Cziesla, S. Eichoff, N. Arts y D. Winter (eds.): The Big Puzzle. Holos. Bonn: 143-163.

Bórmida, M. (1969): "El Puntarrubiense". Trabajos de Prehistoria, XXVI: 7-116. Madrid.

- (1973): "Ergon y mito. Una hermenéutica de la cultura material de los Ayoreo del Chaco Boreal". Scripta Ethnologica, 1 (1): 9-68.

BouRKe, J. (1891): "Arrows and Arrow-makers. Remarks". American Anthropologist, 4: 71-74.

CABrera, G.; Franky, C. y MAhecha, D. (1994): Aportes a la Etnografía de los Nukak y su lengua-Aspectos sobre Fonología Segmental-. Trabajo de Grado para optar al título de Antropólogo. Facultad de Ciencias Humanas Universidad Nacional, Santafé de Bogotá. Colombia.

Cárdenas, D. y Politis, G. (e.p.): “Territorio, movilidad, etnobotánica y manejo del bosque de los Nukak orientales (Amazonia, Colombia)". Boletín del Museo del Oro. Santafé de Bogotá, Colombia.

Ceresole, G. y Slavsky, J. (1985): "Informe preliminar sobre la Localidad Lobería 1 (Pcia. de Buenos Aires)". VIII Congreso Nacional de Arqueología Argentina. Resúmenes de los Trabajos (Concordia, 1985): 1-4.

Chamberlain, A. (1997): "Commentary: Missing stages of life - towards the perception of children in archaeology". En J. Moore y E. Scott (eds.): Invisible People and Processes. Writing Gender and Childhood into European Archaeology. Leicester University Press. Londres: 248-250.

Chaves, M. y Wirpsa, L. (1988): "Aparecen los Nukak". Noticias Antropológicas, 89.

ClaAssen, C. (ed.) (1992): Exploring GenderThroughArchaeology. Prehistory Press. Madison, Wisconsin.

Clastres, P. (1972): Chroniques des indiens Guayaki. Libraire Plon. Francia.

Conkey, M. y Spector, J. (1984): “Archaeology and the stu- dy of gender". En M. Schiffer (ed.): Advances in Archaeological Method and Theory, 7. Academic Press. Nueva York: 1-38.

De Aparicio, F. (1932): “Contribución al estudio de la arqueología del litoral atlántico de la Provincia de Buenos Aires". Boletín de la Academia Nacional de Ciencias de Córdoba, XXXII: 1-180.

Dawe, B. (1997): "Tiny Arrowheads: Toys in the Toolkit". Plains Anthropologist, 42 (161): 303-318.

DrAPER, P. (1976): "Social and economic constraints on child life among the !Kung". En R. Lee y I. De Vore (eds.): Kalahari hunter-gatherers. Harvard University Press. Cambridge: 199-210.

Draper, P. y Cashdan, E. (1989): "Technological change and child behaviour among the !Kung”. Ethnology, 27 (4): 339-365.

Flegenheimer, N. (1991): "Bifacialidad y piedra pulida en sitios pampeanos tempranos". Shincal, 3: 64-78.

- (1995). "The hill top of Cerro El Sombrero, Argentina". Current Research in the Pleistocene, 12: 11-13.

Flegenheimer, N. y Zárate, M. (1997): "Considerations on Radiocarbon and Calibrated Dates from Cerro la China and Cerro el Sombrero, Argentina". Current Research in the Pleistocene 12: 11-13.

FINLAY, N. (1997): "Kid knapping: the missing children in lithic analysis". En J. Moore y E. Scott (eds.): Invisible People and Processes. Writing Gender and Childhood into European Archaeology. Leicester University Press. Londres: 203-212.

Fisher, A. (1990): "On being a pupil flintknapper of 11,000 years ago". En E. Cziesla, S. Eichoff, N. Arts y D. Winter (eds.): The Big Puzzle. Holos. Bonn: 447-464.

Franky, C.; Cabrera, G. y Mahecha, D. (1995): Demografía y movilidad socio-espacial de los Nukak. Documento de Trabajo, 2. Fundación GaiaAmazonas. Santafé de Bogotá, Colombia.

FrISON, G. (1970): "The Kobold site, 24BH406: A postAltithermal Record of Buffalo Jumping for the Northwestern Plains". Plains Anthropologist, 15 (47): 1-35.

Gero, J. (1991): "Genderlithics: Women's Roles in Stone Tool Production”. En J. Gero y M. Conkey (eds.): Engendering Archaeology. Women in Prehistory. Blackwell. Oxford: 163-193.

Gero, J. y ConKey, M. (eds.) (1991): Engendering Archaeology. Women in Prehistory. Blackwell. Oxford.

GoNZÁLEZ, A.R. (1953): "La boleadora. Sus áreas de dispersión y tipos". Revista del Museo de la Universidad de La Plata (N.S.) IV. Sección Antropología: 133-292.

Gradin, C. (1981-1982): "Las pinturas de la Cueva Grande (Arroyo Feo). Área Río Pinturas-Provincia de Santa Cruz". Relaciones, XV: 241-265.

Gusinde, M. (1983): Los indios de Tierra del Fuego. Centro Argentino de Etnología Americana. Buenos Aires.

Hammond, G. y Hammond, N. (1981): "Child's play: a distorting factor in archaeological distribution". American Antiquity, 46 (3): 834-836.

T. P., 55, n. ${ }^{\circ} 2,1998$ 
Hawkes, K.; O'Connell, J.F. y Blurton Jones, N.G. (1995): "Hazda Children's Foraging: Juvenile Depedency, Social Arrangements, and Mobility among Hunter-Gatherer". Current Anthropology, 16 (4):688-700.

Holmberg, A (1978): Nómadas del arco largo. Los sirionó del oriente boliviano. Instituto Indigenista Interamericano. Ediciones Especiales, 77. México.

James, A. y Prout, A. (1990): Constructing and Reconstructing Childhood. The Farmel Press. Basingtocke.

LEIPUS, M. (1997): “Morfología y función de los artefactos líticos del sitio Arroyo Seco 2, Pdo. de Tres Arroyos, Pcia. de Buenos Aires". Resúmenes del XII Congreso Nacional de Arqueología Argentina. La Plata.

Lillehammer, G. (1989): "A Child is Born. The Child's World in an Archaeological Perspective". Norwigian Archaeological Review, 22 (2): 89-105.

Madrid, P. y Politis, G. (1991):"Estudios paleoambientales en la Región Pampeana: un enfoque multidisciplinario". Actas del XI Congreso Nacional de Arqueología Chilena (Santiago de Chile, 1988), I. Santiago de Chile: 131-152.

MAdRID, P. y SAlEmme, M. (1991): "La ocupación tardía del sitio 1 de la Laguna Tres Reyes, Pdo. de Adolfo Gonzáles Chaves, Pcia. de Buenos Aires". Boletín del Centro, 3: 165-179.

Madrid, P.; Politis, G.; Leipus, M., y Landini, C. (1991): "Estado actual de las investigaciones en el sitio 1 de Laguna Tres Reyes: análisis lítico tecno-morfológico y procesos de formación del sitio". Boletín del Centro, 2: 112-122.

Metraux, A. (1948): "The hunting and gathering people of the Rio Negro Basin". Handbook of Southamerican Indians, 3: 861-867. Smithsonian. Washington.

Mondragón, H. (1990): Estudio para el establecimiento de un programa de defensa de la comunidad indígena Nukak. Informe presentado al Programa de Rehabilitación Nacional (PNR) de la Presidencia de la República de Colombia. Santafé de Bogotá.

Morre, J. y Scott, E. (eds.) (1997): Invisible People and Processes. Writing Gender and Childhood into EuropeanArchaeology. Leicester University Press. Londres.

Musters, G. (1997): Vida entre los Patagones. El Elefante Blanco. Buenos Aires.

Nami, H. (1994): "Paleoindio, Cazadores-Recolectores y Tecnología Lítica en el Extremo Sur de Suramerica Continental". En J.L. Lanata y L.A. Borrero: Arqueología de Cazadores-recolectores. Límites, Casos y Aperturas. Arqueología Contemporánea. Buenos Aires: $89-104$.

Reichel-Dolmatoff, G. (1967): “ A brief report on urgent ethnological research in the Vaupes area. Colombia, South America". Bulletin of International Committee on Urgent Anthropological Research, 9: 53-62.

REID, H. (1979): Some aspects of movement, growth, and change among the Hupdu Makú indians of Brazil. Ph.D. Dissertation. Cambridge University. Cambridge.

ReinA, L. (1990): "Actividades relacionadas con los Nukak". Mopa-Mopa, 5: 17-25.

Politis, G. (1984): Arqueología del Area Interserrana Bonaerense. Tesis Doctoral. Facultad de Ciencias Naturales y Museo. Universidad Nacional de La Plata, Argentina.

- (1989): “Quién mató al megaterio". Ciencia Hoy, 1 (2): 26-35.

- (1991): "Fishtail projectil points in the Southern Cone of South America: an overview". En R. Bonnichsen y K. Turnmire (eds.): Clovis Origins and Adaptations. Center for the Study of the First Americans. Oregon, EEUU: 287-301.

- (1992): "La Arquitectura del Nomadismo en la Amazonía Colombiana”. Proa, 412: 11-20.

- (1996a): "Moving to produce. Nukak mobility and settlement patterns inAmazonia". WorldArchaeology, 27: 492-511.

- (1996b): Nukak. Instituto Amazónico de Investigaciones Científicas SINCHI. Santafé de Bogotá. Colombia

- (e.p.): "La formación de sitios de cazadores-recolectores en las Tierras Bajas Sudamericanas: un caso de estudio etnoarqueológico". En A. Durán y R. Bracco (eds.): Arqueología de Tierras Bajas Suramericanas. Ministerio de Educación y Cultura. Montevideo. Uruguay.

Politis, G. y Rodríguez, J. (1994): “Algunos aspectos de la subsistencia de los Nukak de la Amazonía Colombiana”. Colombia Amazónica, 7 (1-2): 169-207.

Politis, G.; Martínez, G. y Rodríguez, J. (1997): "Caza, recolección y pesca como estrategia de explotación de recursos en forestas tropicales lluviosas: los Nukak de la amazonía colombiana". Revista Española deAntropología Americana, 27: 167-197.

ScOTT, E. (1997): "Introduction: On the incompletness of archaeological narratives". En J. Moore y E. Scott (eds.): Invisible People and Processes. Writing Gender and Childhood into European Archaeology. Leicester University Press. Londres: 1-12.

Silverwood-Cope, P. (1972): A contribution to the ethnography of the Colombian Makú. Ph. D. Dissertation. Cambridge University. Cambridge.

Torres, W. (1994): "Nukak: aspectos etnográficos". Revista Colombiana de Antropología, 31: 197-234.

Weismer, T. y Gallimore, R. (1977): "My Brother's Keeper. Child and Sibling Caretaking". Current Anthropology, 18: 160-190.

WIESSNER, P. (1983): "Style and ethnicity in the Kalahari San projectile point". American Antiquity, 50: 253-276.

WILK, R. y SCHIFFER, M. (1979): "The archaeology of vacant lots in Tucson, Arizona". American Antiquity, 44: 530-536.

T. P., 55, n. ${ }^{\circ} 2,1998$ 